\title{
Ferromagnetic Behaviors in Fe-Doped NiO Nanofibers Synthesized by Electrospinning Method
}

\author{
Yi-Dong Luo, ${ }^{1}$ Yuan-Hua Lin, ${ }^{1}$ Xuehui Zhang, ${ }^{1}$ Deping Liu, ${ }^{2}$ Yang Shen, ${ }^{1}$ and Ce-Wen Nan ${ }^{1}$ \\ ${ }^{1}$ State Key Laboratory of New Ceramics and Fine Processing, Department of Materials Science and Engineering, Tsinghua University, \\ Beijing 100084, China \\ ${ }^{2}$ Department of Cardiology, Beijing Hospital, The Ministry of Health, Beijing 100730, China
}

Correspondence should be addressed to Yuan-Hua Lin; linyh@mail.tsinghua.edu.cn

Received 5 October 2012; Revised 4 December 2012; Accepted 18 December 2012

Academic Editor: Zhengren Huang

Copyright (C) 2013 Yi-Dong Luo et al. This is an open access article distributed under the Creative Commons Attribution License, which permits unrestricted use, distribution, and reproduction in any medium, provided the original work is properly cited.

\begin{abstract}
$\mathrm{Ni}_{1-x} \mathrm{Fe}_{x} \mathrm{O}$ nanofibers with different $\mathrm{Fe}$ doping concentration have been synthesized by electrospinning method. An analysis of the phase composition and microstructure shows that $\mathrm{Fe}$ doping has no influence on the crystal structure and morphology of $\mathrm{NiO}$ nanofibers, which reveals that the doped $\mathrm{Fe}$ ions have been incorporated into the NiO host lattice. Pure NiO without Fe doping is antiferromagnetic, yet all the $\mathrm{Fe}$-doped $\mathrm{NiO}$ nanofiber samples show obvious room-temperature ferromagnetic properties. The saturation magnetization of the nanofibers can be enhanced with increasing Fe doping concentration, which can be ascribed to the double exchange mechanism through the doped Fe ions and free charge carriers. In addition, it was found that the diameter of nanofibers has significant impact on the ferromagnetic properties, which was discussed in detail.
\end{abstract}

\section{Introduction}

Diluted magnetic semiconductors (DMS) have been intensively studied due to their high potential for applications in spin-dependent semiconductor electronics [1]. Practical spintronic materials should have high Curie temperatures, high spin polarization of charge carriers, and compatibility with semiconductors $[2,3]$. Lots of experiments were carried out to study the fascinating properties of the oxide-based DMSs (e.g., $\mathrm{ZnO}, \mathrm{TiO}_{2}, \mathrm{SnO}_{2}, \mathrm{In}_{2} \mathrm{O}_{3}$, etc.) with various transition metal (TM) ions doped [4-7]. But, compared to the TM-doped oxide-based materials mentioned above, it is more feasible to realize the p-type doping in $\mathrm{NiO}$ system.

Recently, one-dimensional nanofibers have received intensive attention due to their excellent magnetic, optical, electric, and chemical properties [8]. The low-symmetry structure will affect their peculiar magnetic properties [9]. In the recent study of DMS materials, nanoparticles, films structure of $\mathrm{NiO}$ have already been prepared by sol-gel techniques, hydrothermal route, and Pulsed lase deposition [10-12]. Unfortunately, the investigation of the Fe-doping effect on ferromagnetism of one-dimensional DMS fibers is very limited. Thus, it is necessary to study the influence of low dimensional structure on NiO-based system. Among all the methods of making one-dimensional nanostructure magnetic materials, electrospinning is a simple, versatile, and convenient approach with the characteristic of easy control and low cost [13].

Our previous works have already reported the roomtemperature $\mathrm{FM}$ behavior of the $\mathrm{Fe}$-doped $\mathrm{NiO}$ nanoparticles $[12,14]$. Although pure $\mathrm{NiO}$ exhibits insulating character and antiferromagnetic order at room temperature, introduction of $\mathrm{Fe}$ ions will break the symmetry of the system in $\mathrm{NiO}$, showing the room-temperature ferromagnetism $[15,16]$. Therefore, it is anticipated that Fe-doped $\mathrm{NiO}$ nanofiber would exhibit ferromagnetism.

In this work, we prepared $\mathrm{Fe}$-doped $\mathrm{NiO}$ nanofibers (NFO) and observed remarkable FM properties at room temperature. The result may be attributed to the double exchange mechanism through the doped $\mathrm{Fe}$ ions and free charge carriers.

\section{Experimental Procedure}

2.1. Preparation of Fe-doped Nanofibers. $\mathrm{Ni}(\mathrm{AC})_{2} \cdot 4 \mathrm{H}_{2} \mathrm{O}$ and the appropriate amount of $\mathrm{Fe}\left(\mathrm{NO}_{3}\right)_{3} \cdot 9 \mathrm{H}_{2} \mathrm{O}$ with different 


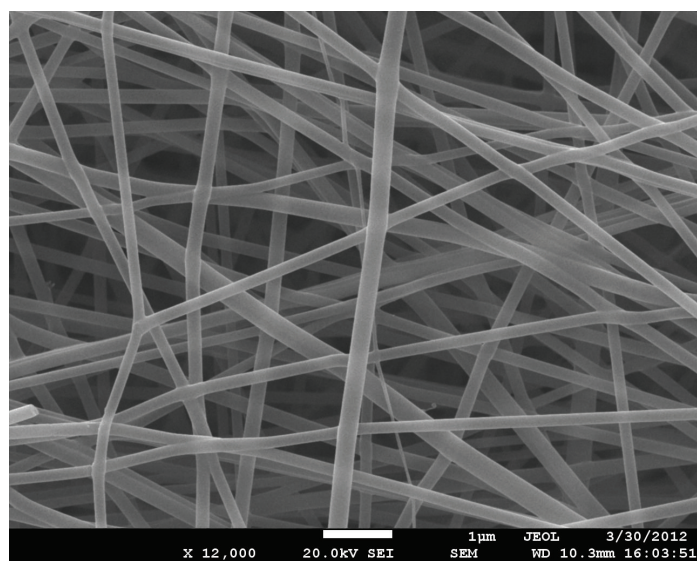

(a)

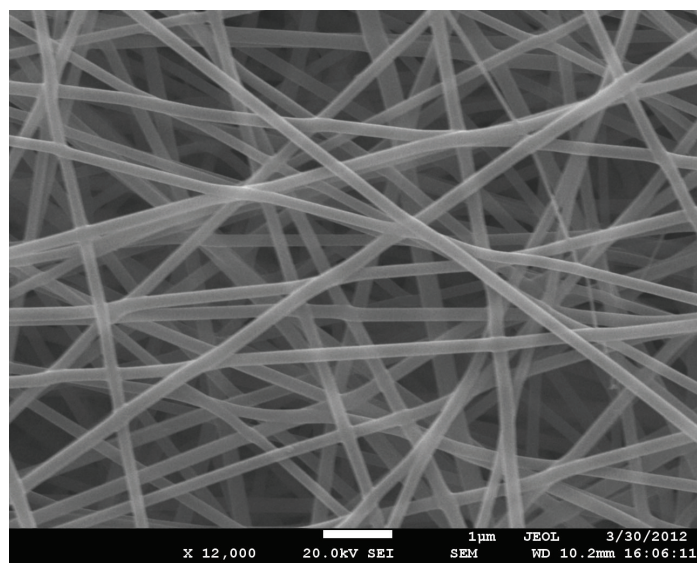

(c)

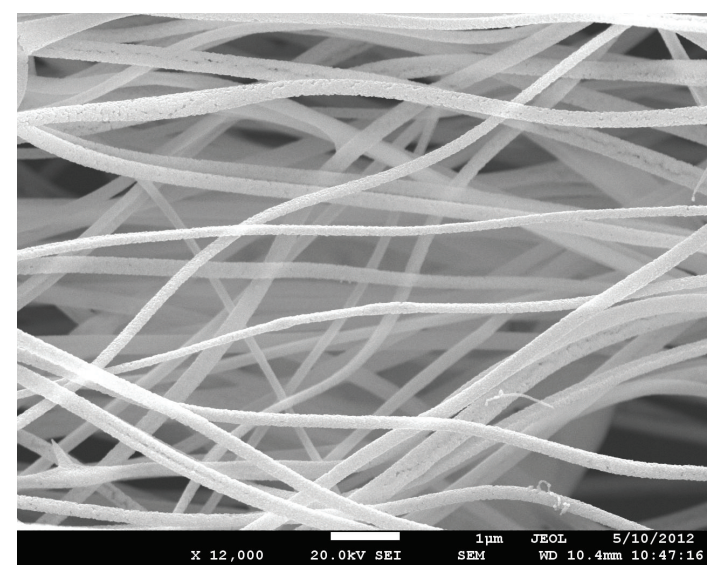

(b)

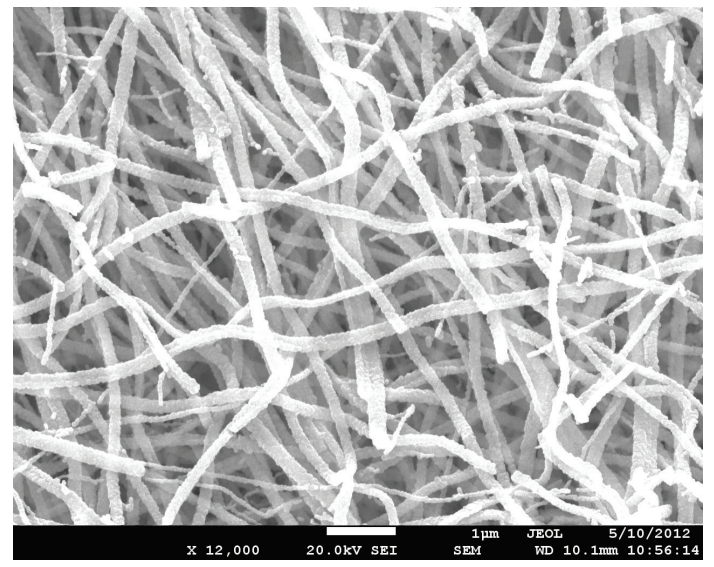

(d)

FIGURE 1: (a) SEM images of the undoped NiO/PVP nanofibers before calcination. (b) SEM images of the undoped NiO/PVP nanofibers after calcination. (c) SEM images of the Fe-doped NiO/PVP composite nanofibers before calcination. (d) SEM images of the Fe-doped NiO/PVP composite nanofibers after calcination.

atomic ratios were dropped slowly into the mixed solution (made by $45 \mathrm{~mL}$ alcohol and $5 \mathrm{~mL}$ water) with stirring. Then $2 \mathrm{~g}$ PVP powder were added slowly into the solution under stirring, and the sol solution was obtained for electrospinning. The precursor sol solution was loaded into a $10 \mathrm{~mL}$ plastic syringe with a syringe needle of which the internal diameter is $0.5 \mathrm{~mm}$. The needle was connected to a DC high-voltage power supply. In our experiment, a voltage of $12 \mathrm{kV}$ was applied between the cooper plate collector and the syringe needle with a distance of $12 \mathrm{~cm}$. The $\mathrm{PVP} / \mathrm{Ni}\left(\mathrm{CH}_{3} \mathrm{COO}\right)_{2}$ composite nanofibers were collected on the cooper plate during electrospinning processes. Pure $\mathrm{NiO}$ and $\mathrm{Fe}$-doped $\mathrm{NiO}$ nanofibers were finally obtained by calcination at $660^{\circ} \mathrm{C}$ for $3 \mathrm{~h}$ in air to remove PVP completely.

2.2. Characterization. X-ray diffraction (XRD) was employed to investigate the crystal structure of nanofibers. And the morphologies of nanofibers were characterized by scanning electron microscope (SEM). The valence state of the Fe ions was analyzed by X-ray photoelectron spectroscopy (XPS) and magnetic properties of the samples were measured by Physical Property Measurement System (PPMS).

\section{Results and Discussion}

Figure 1 shows the SEM images of the nanofibers before and after calcination. Figures 1(a) and 1(c) show the SEM images of undoped NiO/PVP and Fe-doped $\mathrm{NiO} / \mathrm{PVP}$ composite nanofibers with smooth surface before calcination. They are several millimeters long with a diameter of approximate $270 \mathrm{~nm}$. Furthermore, in contrast to pure NiO/PVA composite nanofibers, the doping $\mathrm{Fe}$ ions in $\mathrm{NiO}$ do not influence the morphologies of doped samples. As shown in Figures $1(\mathrm{~b})$ and $1(\mathrm{~d})$, after calcined at $660^{\circ} \mathrm{C}$, the diameters of all NiO-based nanofibers shrank drastically to 60-100 nm due to the decomposition of PVP and the transformation from metal salts to metal oxides. Figure 2 shows the XRD patterns of various $\mathrm{NiO}$-based nanofiber samples after calcination. Obviously, all of these samples are pure cubic crystalline $\mathrm{NiO}$ phase, and no impurity phase appears.

The existence of the Fe ions in the present NFO nanofibers was verified by the XPS measurements as shown in Figure 3. It can be seen that the spectra consist of Fe $2 \mathrm{p}_{3 / 2}(713.2 \mathrm{eV})$ and $2 \mathrm{p}_{1 / 2}(724.5 \mathrm{eV})$ peaks. The chemical state of the Fe ions may be a mixture of $\mathrm{Fe}^{2+}$ and $\mathrm{Fe}^{3+}$ as seen from the broad peaks of 


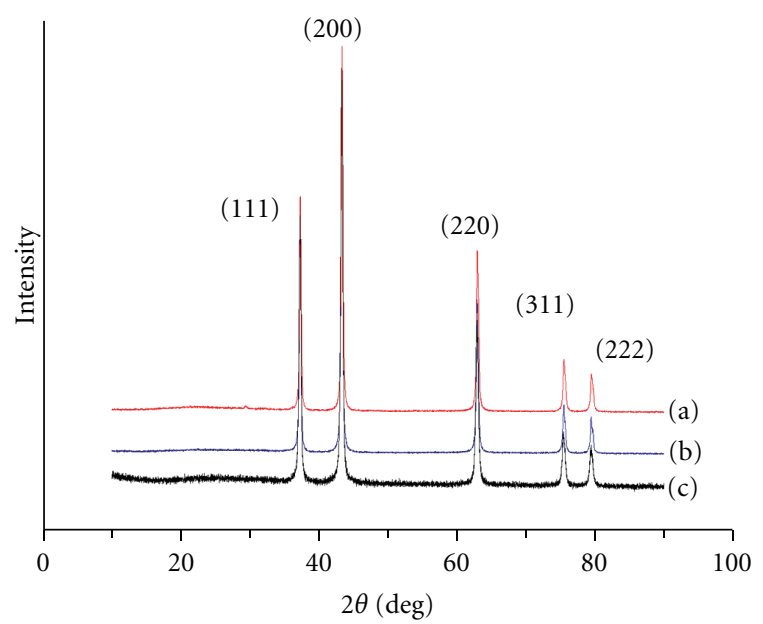

FIGURE 2: XRD patterns of various nanofibers made by electrospinning after calcination. (a) $3 \% \mathrm{Fe}$, (b) $5 \% \mathrm{Fe}$, (c) $0 \% \mathrm{Fe}$.

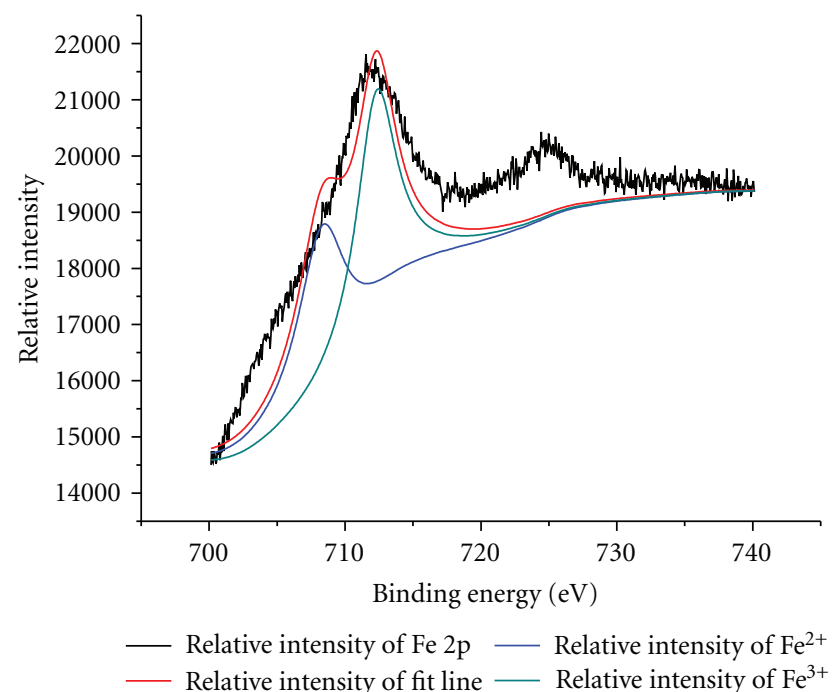

FIgure 3: Core-level XPS spectra of $\mathrm{Fe} 2 \mathrm{p}$ for $\mathrm{Ni}_{0.97} \mathrm{Fe}_{0.03} \mathrm{O}$ nanofibers and XPS simulation for Fe $2 p$ spectra.

Fe 2p. As previously reported [17], in the Fe-doped oxidesbased DMS systems, the possible presence of a secondary phase (e.g., metallic magnetic Fe particles) can also be the origin of ferromagnetism. However, no metallic $\mathrm{Fe}^{0}\left(2 \mathrm{p}_{3 / 2}\right.$ binding energy $\sim 706 \mathrm{eV}$ and $2 \mathrm{p}_{1 / 2}$ binding energy $\sim 719 \mathrm{eV}$ ) was observed in the present spectra. Therefore, according to the Fe 2p XPS spectra and XRD results, as for our Fe-doped $\mathrm{Ni}_{1-x} \mathrm{Fe}_{x} \mathrm{O}$ nanofibers, suggestion of the secondary Fe metal cluster phase as the origin of ferromagnetism can be ruled out.

As shown in Figure 4, with the increasing of the concentration of the Fe-doping, the samples exhibit obvious FM properties at room temperature. In these type $\mathrm{Fe}$-doped $\mathrm{NiO}$ samples, the $\mathrm{Ni}$ ions are partially substituted by the Fe ions, which are randomly localized over the host lattice. Thus, this kind of disorder certainly breaks the translation symmetry of the system and the original magnetic order in $\mathrm{NiO}$ grains is

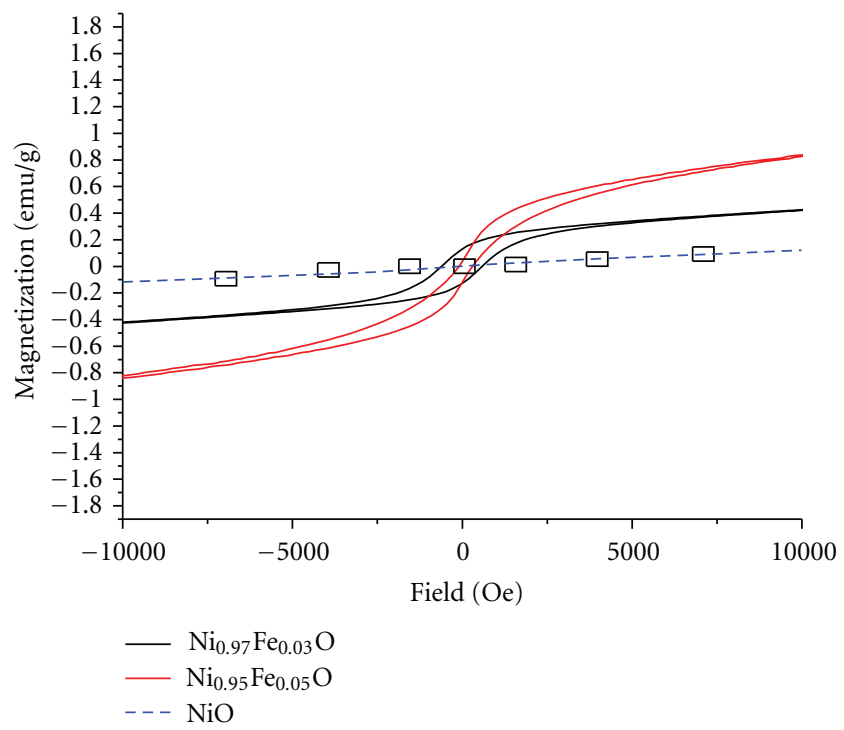

FIgURE 4: Magnetization-field (M-H) loop curves of various NFO nanofibers.

interrupted. The results are similar with the study of the LiFe codoped films [14]. The ferromagnetism in the Fe-doped $\mathrm{NiO}$ could be caused by the double exchange through the introduced magnetic Fe ions and the related defects (e.g., $\left.\mathrm{Fe}_{\mathrm{Ni}}\right)$. That is to say, electrons may weakly trapped in the $\mathrm{Fe}_{\mathrm{Ni}}$ defect site, where the electron occupies an orbital which overlaps the d shells of both $\mathrm{Fe}$ neighbors, enhancing the interaction between $\mathrm{Fe}$ ions, resulting the ferromagnetism of the nanofibers. In addition, with the increase of doped Fe ions, the amount of the magnetic Fe ions and the $\mathrm{Fe}_{\mathrm{Ni}}$ defects will also increase, which will enhance the FM doubleexchange interaction.

Moreover, compared with the results of Liu's group [9], we find the diameter of nanofibers has significant impact on the ferromagnetic properties. We suppose that there are two possible mechanisms of the remarkable FM properties. The first mechanism is associated with the transition of double sublattice to multisublattice states [18]. Yet, this explanation only suits the particles whose diameter is less than $30 \mathrm{~nm}$. The other mechanism is related to the change of the impurity states. With the decreasing size, the impurity states may become much deeper in energy [19], which may enhance the coupling interaction between the $3 \mathrm{~d}$ spins of $\mathrm{Fe}$ ions and the carriers, giving an obvious enhancement of FM properties of the nanofibers. More experiments are carrying on to confirm this assumption.

\section{Conclusions}

In conclusion, nanofibers were prepared by electrospinning method. XPS spectra reveal that the Fe 2p core-level photoemission spectra consisted of the $\mathrm{Fe}^{2+}$ and $\mathrm{Fe}^{3+}$ components, and no metallic $\mathrm{Fe}$ appeared. All the nanofibers exhibited obvious ferromagnetic ordering at room temperature which 
should be ascribed to the double exchange through the introduced magnetic Fe ions and related defects.

\section{Acknowledgments}

This work was supported by the Ministry of Science and Technology of China through a 973 Project under Grant no. 2009CB623303, NSF of China (51025205, 51272181, and 51272121).

\section{References}

[1] H. Ohno, "Making nonmagnetic semiconductors ferromagnetic," Science, vol. 281, no. 5379, pp. 951-956, 1998.

[2] S. A. Wolf, D. D. Awschalom, R. A. Buhrman et al., "Spintronics: a spin-based electronics vision for the future," Science, vol. 294, no. 5546, pp. 1488-1495, 2001.

[3] T. Dietl and H. Ohno, "Engineering magnetism in semiconductors," Materials Today, vol. 9, no. 11, pp. 18-26, 2006.

[4] M. Kobayashi, Y. Ishida, J. L. Hwang et al., "Characterization of magnetic components in the diluted magnetic semiconductor $\mathrm{Zn}_{1-\mathrm{x}} \mathrm{Co}_{\mathrm{x}} \mathrm{O}$ by $\mathrm{x}$-ray magnetic circular dichroism," Physical Review B, vol. 72, no. 20, 4 pages, 2005.

[5] Y. Matsumoto, M. Murakami, T. Shono et al., "Roomtemperature ferromagnetism in transparent transition metaldoped titanium dioxide," Science, vol. 291, no. 5505, pp. 854-856, 2001.

[6] S. B. Ogale, R. J. Choudhary, J. P. Buban et al., "High temperature ferromagnetism with a giant magnetic moment in transparent co-doped $\mathrm{SnO}_{2-\delta}$," Physical Review Letters, vol. 91, no. 7, Article ID 077205, 4 pages, 2003.

[7] J. Philip, A. Punnoose, B. I. Kim et al., "Carrier-controlled ferromagnetism in transparent oxide semiconductors," Nature Materials, vol. 5, no. 4, pp. 298-304, 2006.

[8] F. Qian, S. Gradečak, Y. Li, C. Y. Wen, and C. M. Lieber, "Core/multishell nanowire heterostructures as multicolor, high-efficiency light-emitting diodes," Nano Letters, vol. 5, no. 11, pp. 2287-2291, 2005.

[9] S. H. Liu, J. F. Jia, J. Wang et al., "Synthesis of Fe-doped $\mathrm{NiO}$ nanofibers using electrospinning method and their ferromagnetic properties," Journal of Magnetism and Magnetic Materials, vol. 324, no. 13, pp. 2070-2074, 2012.

[10] S. Manna, A. K. Deb, J. Jagannath, and S. K. De, "Synthesis and room temperature ferromagnetism in $\mathrm{Fe}$ doped $\mathrm{NiO}$ nanorods," Journal of Physical Chemistry C, vol. 112, no. 29, pp. 10659-10662, 2008.

[11] W. Yan, W. Weng, G. Zhang et al., "Structures and magnetic properties of $(\mathrm{Fe}, \mathrm{Li})$-codoped $\mathrm{NiO}$ thin films," Applied Physics Letters, vol. 92, no. 5, Article ID 052508, 3 pages, 2008.

[12] J. Wang, J. Cai, Y. H. Lin, and C. W. Nan, "Room-temperature ferromagnetism observed in Fe-doped NiO," Applied Physics Letters, vol. 87, no. 20, 3 pages, 2005.

[13] M. Zhao, X. Wang, L. Ning et al., "Synthesis and optical properties of Mg-doped $\mathrm{ZnO}$ nanofibers prepared by electrospinning," Journal of Alloys and Compounds, vol. 507, no. 1, pp. 97-100, 2010.

[14] Y. H. Lin, J. Wang, J. Cai et al., "Ferromagnetism and electrical transport in Fe-doped NiO," Physical Review B, vol. 73, no. 19, 4 pages, 2006.
[15] J. Van Elp, H. Eskes, P. Kuiper, and G. A. Sawatzky, "Electronic structure of Li-doped NiO," Physical Review B, vol. 45, no. 4, pp. 1612-1622, 1992.

[16] Y. H. Lin, R. J. Zhao, C. W. Nan, and M. H. Ying, "Enhancement of ferromagnetic properties of $\mathrm{NiO}: \mathrm{Fe}$ thin film by Li doping," Applied Physics Letters, vol. 89, no. 20, 3 pages, 2006.

[17] Y. J. Kim, S. Thevuthasan, T. Droubay et al., "Growth and properties of molecular beam epitaxially grown ferromagnetic Fe-doped $\mathrm{TiO} 2$ rutile films on TiO2(110)," Applied Physics Letters, vol. 84, no. 18, pp. 3531-3533, 2004.

[18] L. P. Li, L. J. Chen, R. Qi, and G. S. Li, "Magnetic crossover of $\mathrm{NiO}$ nanicrystals at room temperature," Applied Physics Letters, vol. 89, no. 13, Article ID 134102, 3 pages, 2006.

[19] X. Huang, A. Makmal, J. R. Chelikowsky, and L. Kronik, "Sizedependent spintronic properties of dilute magnetic semiconductor nanocrystals," Physical Review Letters, vol. 94, no. 23, Article ID 236801, 4 pages, 2005. 

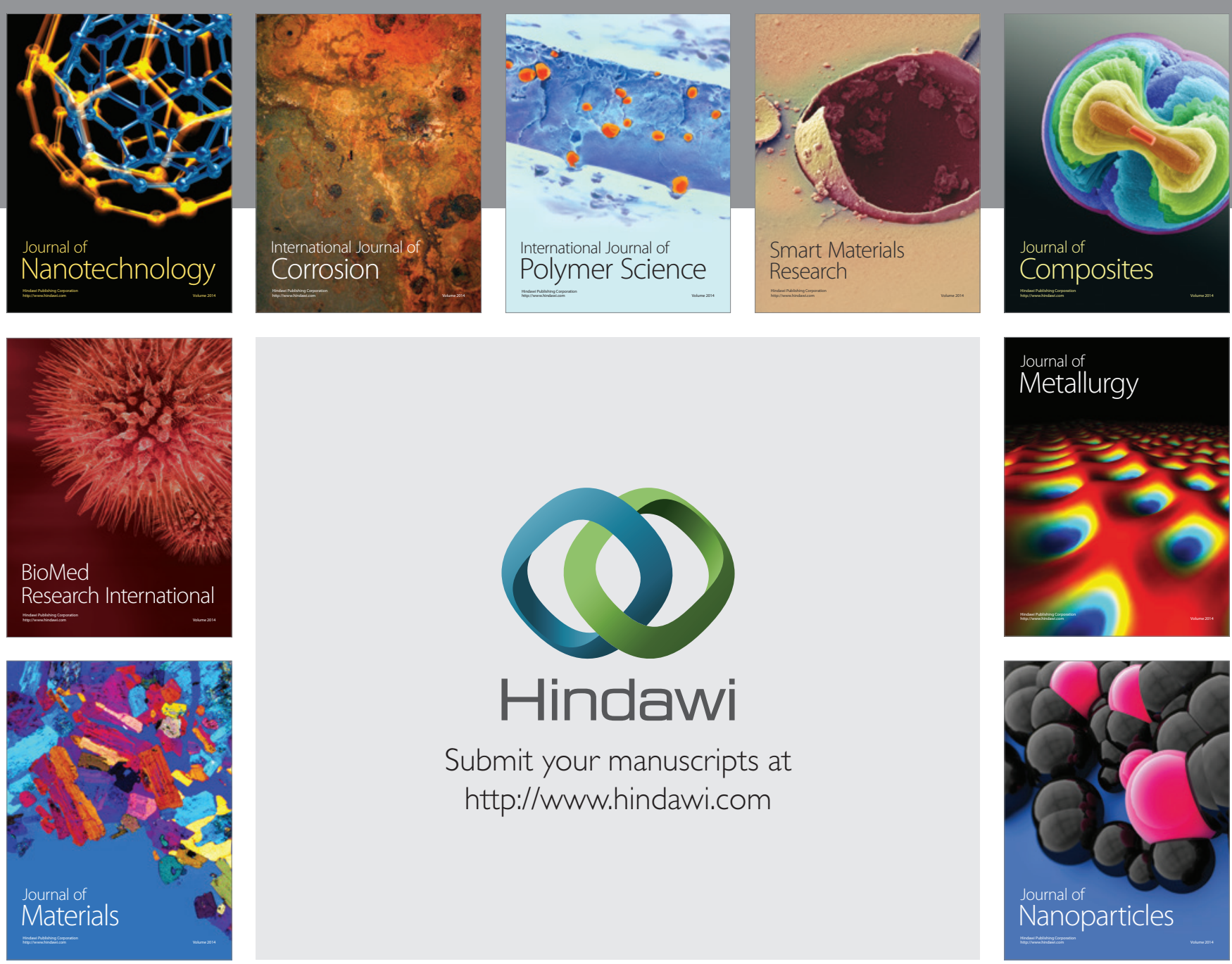

Submit your manuscripts at http://www.hindawi.com
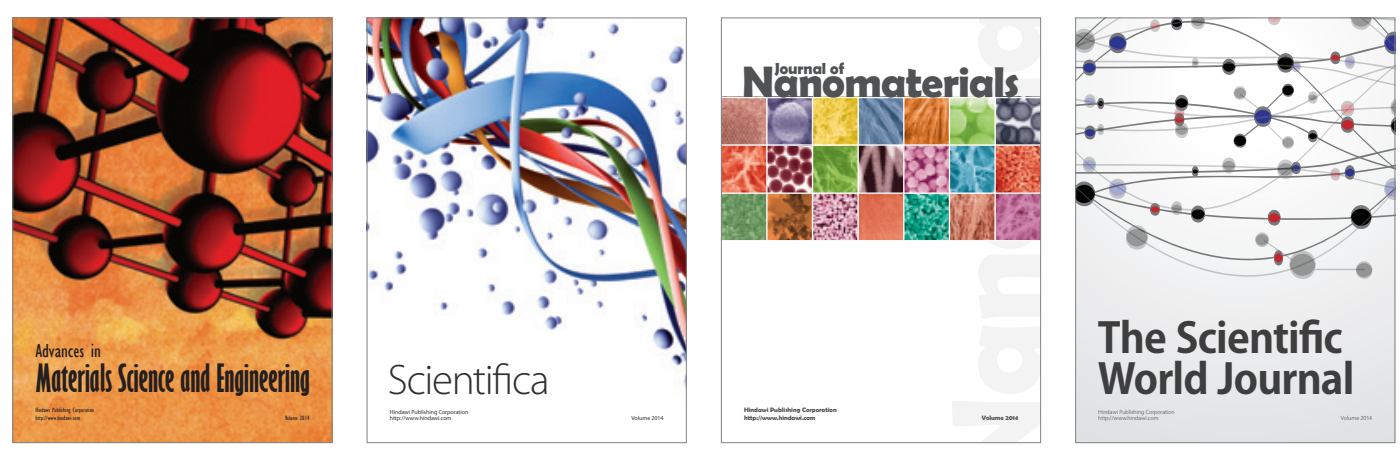

\section{The Scientific World Journal}
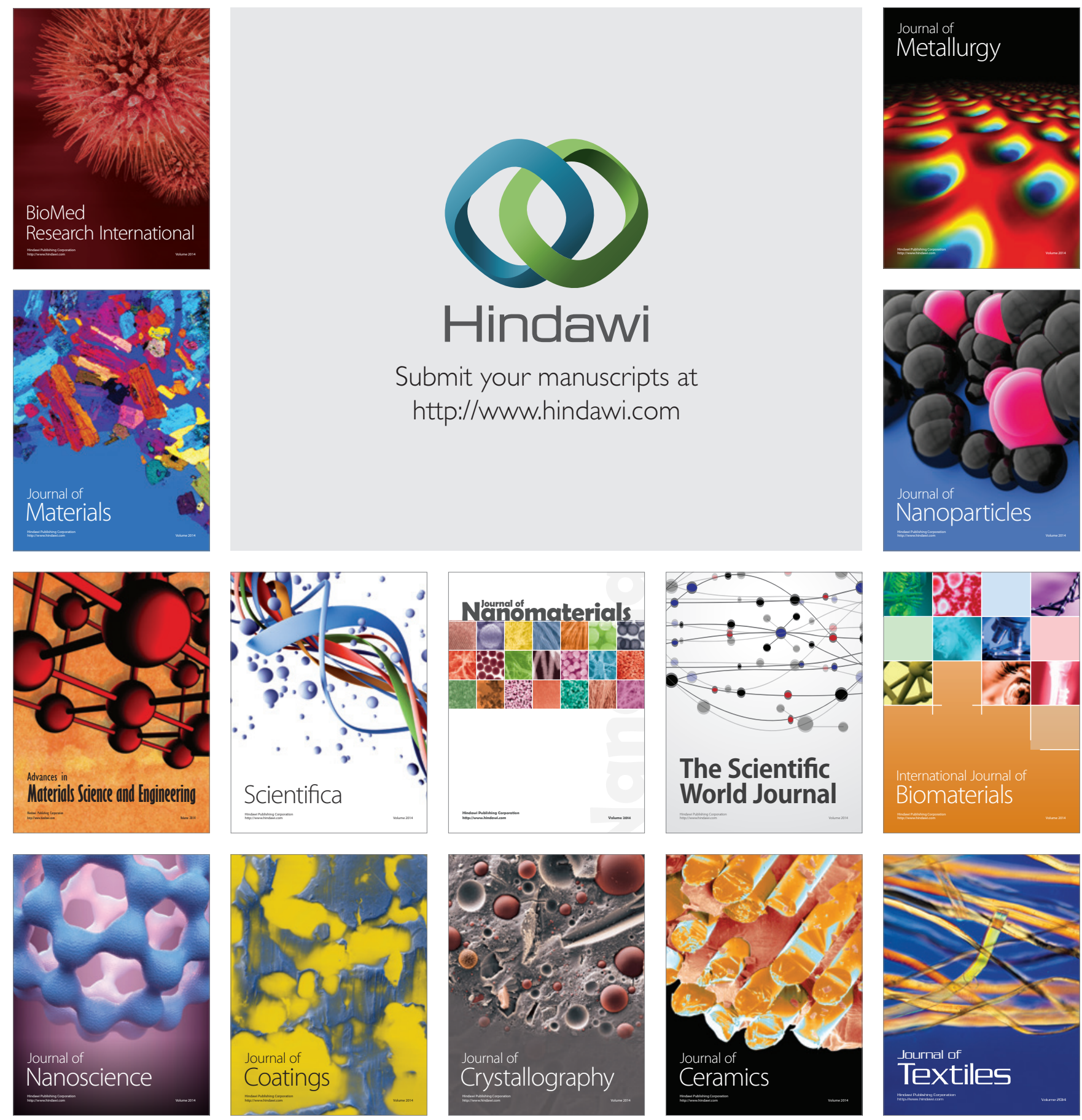